\title{
Numerical representation of the quality measures of triangles and triangular meshes
}

\author{
J. Sarrate, J. Palau and A. Huerta* \\ Departament de Matemàtica Aplicada III, E.T.S. de Ingenieros de Caminos, Canales y Puertos, Universitat \\ Politècnica de Catalunya, Jordi Girona 1-3, E-08034 Barcelona, Spain.
}

\section{SUMMARY}

In this note a new procedure to represent the quality measure for triangles is proposed. The triangles are identified by their three angles and are represented in a bounded domain, called angle representation region, according to the area coordinates, which are common and well-known by finite element users. The developed representation can also be used in order to visualize the characteristics of any quality measure. This new procedure is extended to graphically represent triangular meshes in the angle representation region.

KEY WORDS: Finite element meshes, triangular elements, quality measure.

\footnotetext{
${ }^{*}$ Correspondence to: Departament de Matemàtica Aplicada III, E.T.S. de Ingenieros de Caminos, Canales y Puertos, Universitat Politècnica de Catalunya, Jordi Girona 1-3, E-08034 Barcelona, Spain. E-mail: antonio.huerta@upc.es

Contract/grant sponsor: Ministerio de Educación y Cultura; contract/grant number: DPI2001-2204

Contract/grant sponsor: Ministerio de Educación y Cultura; contract/grant number: REN2001-0925-C03-01
} 


\section{INTRODUCTION}

Unstructured triangular meshes have been extensively used in bi-dimensional analysis over the last decades, see references $[1,2]$ for a detailed review on mesh generation algorithms. It is well known that the error of the finite element analysis depends, among other factors, on the shape of the generated elements. Therefore, all algorithms focus on identifying degenerate triangles (see reference [1] for a classification of triangles according to its shape).

A wide range of measures of quality of triangular elements can be found in the literature

$[1,3,4,5]$. According to [4], a fair measure of the quality of a triangle should clearly identify
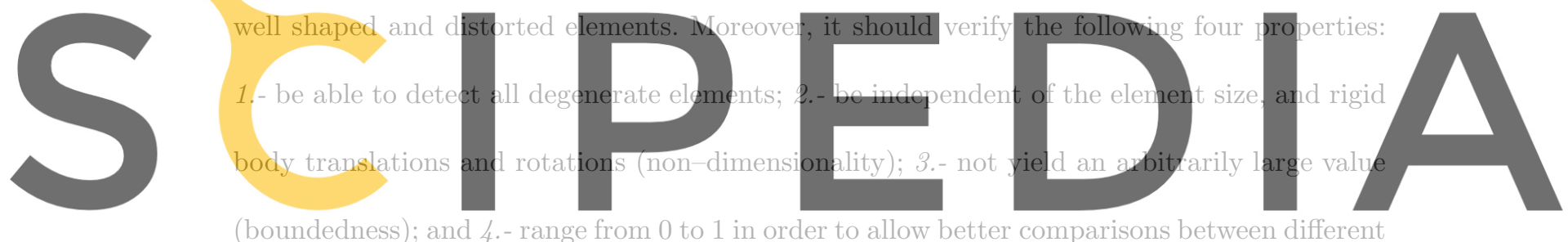

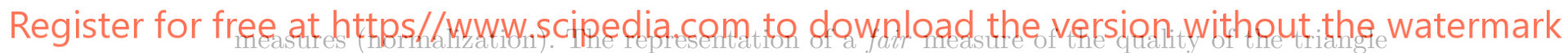

has to highlight these properties. Moreover, it has to visualize and clearly distinguish the well and bad shaped triangles.

[4] introduces the concept of the Universal Similarity Region (USR). In this representation the quality measure of a triangle and/or the triangle itself can be represented in a bounded region. It is important to note that in the USR representation, each family of triangles (a triangle and all the triangles similar to it) is identified by the length of an edge and two angles.

In this paper a new representation of the quality measures of the triangles is presented. It 
identifies each family of triangles by its three inner angles. Moreover, it allows to represent all the triangles in a bounded region, called angle representation region (ARR). In this region, contour levels of a quality measure can easily be plotted. Moreover, the developed representation can be efficiently used in order to compare quality measures. And since it is based on the area coordinates, which are well known by the finite element community, insightful conclusions can be gained about contours depicting measures of quality and about quality measures of specific triangles or meshes.

Several quality measures of a triangle have been developed over the past decades $[3,1,4,5]$. The objective here is not to analyze and compare them. However, some of them will be used to visualize the applicability of the developed representation. In particular, we will use the following measures:
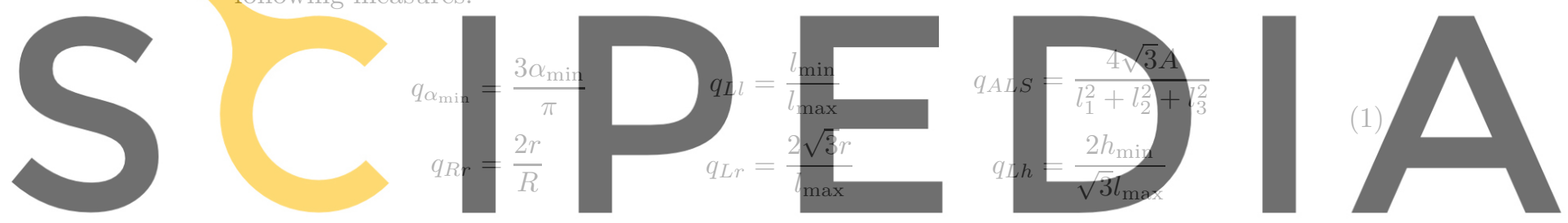

where $\alpha_{\min }$ is the smallest inner angle; $l_{\min }$ and $l_{\max }$ are the length of the shortest and longest

Register for free at https//www.scipedia.com to download the version without the watermark edge respectively; $l_{1}, l_{2}$ and $l_{3}$ are the length of the three sides of the triangle; $A$ is the area of

the triangular element; $r$ is the inradius of a triangle; $R$ is the circumradius of a triangle; and $h_{\min }$ is the minimum height of the triangle.

\section{REPRESENTATION OF A QUALITY MEASURE OF TRIANGLES}

\subsection{The angle representation region}

Since a fair measure of quality of triangles should be independent of the element size, rigid body translations and rotations, the new representation is based on the angles of the triangles, 


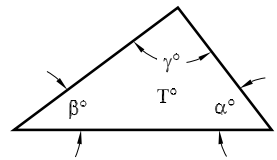

(a)

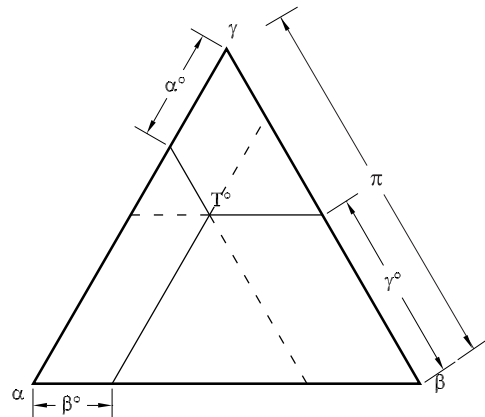

(b)

Figure 1. (a) A given triangle $T^{0}\left(\alpha^{0}, \beta^{0}, \gamma^{0}\right)$ and (b) its

$$
\alpha+\beta+\gamma=\pi
$$
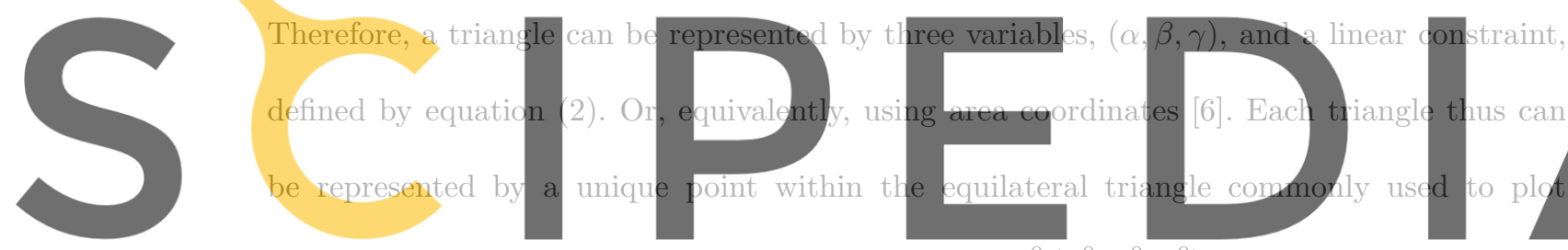

area coordinates. Figure 1.a shows a given triangle $T^{0}\left(\alpha^{0}, \beta^{0}, \gamma^{0}\right)$, and figure 1.b shows its

\section{Register for free at https//www scipedia.com to download the version without the watermark}

The basic triangles can be easily identified in the angle representation region (ARR). The equilateral triangle corresponds to the point $(\alpha, \beta, \gamma)=(\pi / 3, \pi / 3, \pi / 3)$ (see figure 2.a). The isosceles triangles, $\alpha$ being the different angle, are placed on the bisecting line of vertex $\alpha$ (see segment $\overline{\alpha \alpha^{\prime}}$ in figure 2.b). The same property applies for isosceles triangles, where the different angle is $\beta$ or $\gamma$. Note that these three bisecting lines divide the ARR in six equal (or equivalent) regions. Degenerate triangles are placed on the boundary of the ARR. For instance, triangles with $\alpha=\pi$ are represented at vertex $\alpha$. Moreover, if $\alpha$ tends to zero, the representation of the triangle tends to the edge $\overline{\beta \gamma}$. 


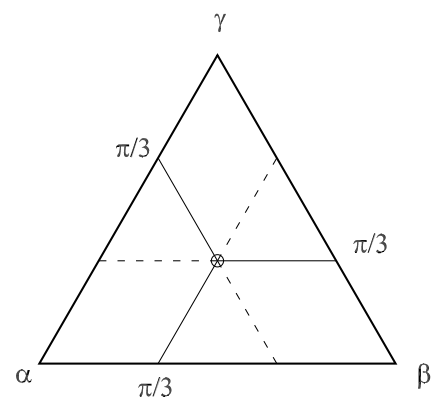

(a)

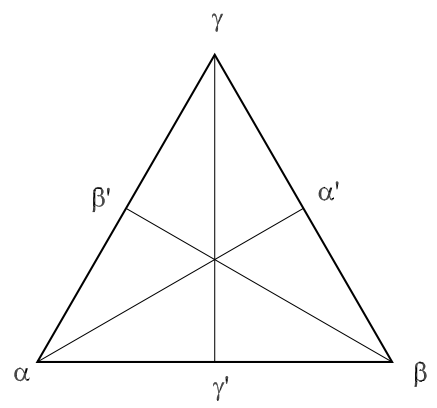

(b)

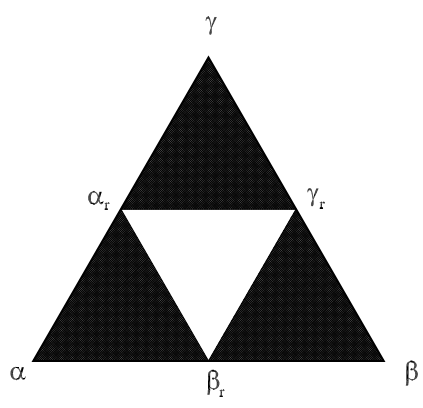

(c)

Figure 2. (a) Representation of an equilateral triangle, (b) Representation of isosceles triangles, (c) Partition of the ARR in two disjoint regions corresponding to triangles with an obtuse inner angle (shadowed region) and triangles with only acute inner angles (white region).

It is straightforward to identify the regions corresponding to acute and obtuse angles in
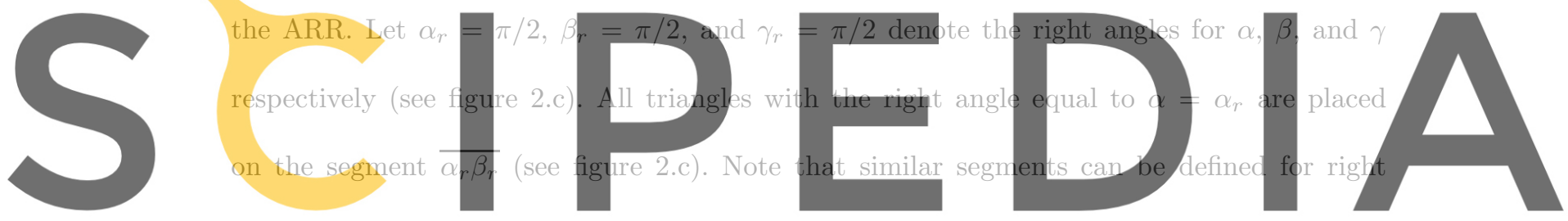

angles $\beta_{r}$ and $\gamma_{r}$. Therefore, the ARR can be subdivided in two disjoint regions. The first

\section{Register for free at https/Awwwscipedia.com to download the version without the watermark}

second region corresponds to triangles with only acute inner angles (white region in figure 2.c).

\subsection{Representation of a measure of quality}

It is extremely simple to represent a measure of the quality of a triangle in the ARR. Given a quality measure, for each triangle (for each point on the ARR) a scalar value can be computed. Thus, it is possible to draw contour levels of this measure of quality. The contour level of any fair measure of the quality of a triangle has to meet the following three conditions in terms of its representation in the ARR: 


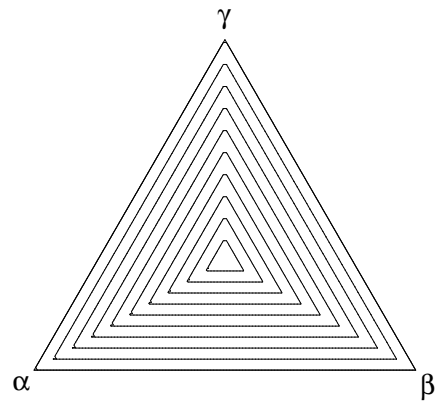

(a)

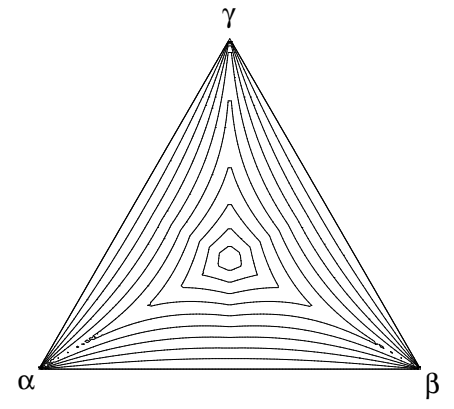

(b)

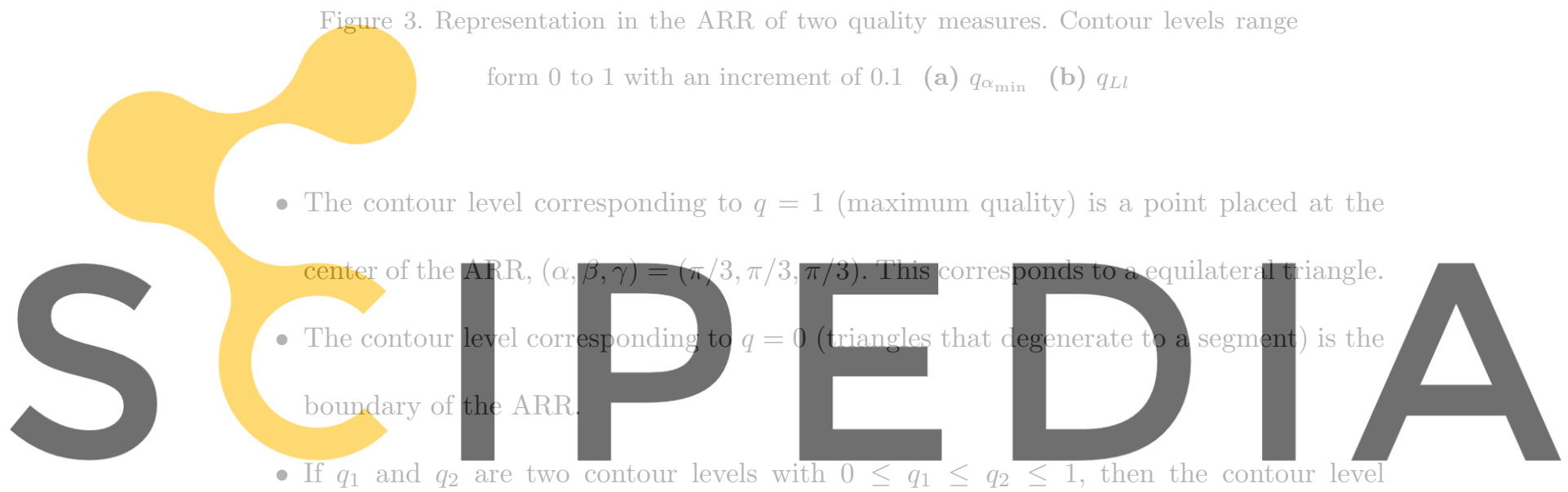

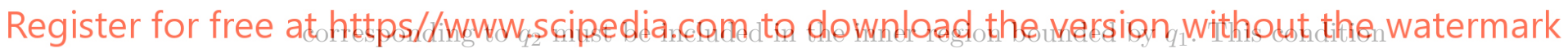

implies that any fair measure will decrease monotonically from the center (the optimal

triangle) to the boundary (degenerate triangles) of the ARR.

Figure 3.a shows the contour levels corresponding to the measure of quality $q_{\alpha_{\min }}$, see (1). In this case, all previous conditions are fulfilled. The contour levels corresponding to the measure of quality $q_{L l}$ are plotted in Figure 3.b. Note that now, the previous conditions are not met. Degenerate triangles with an inner angle equal to $\pi$ have a quality measure different from zero. That is, contour levels converge to cusp at the corners of the ARR. 


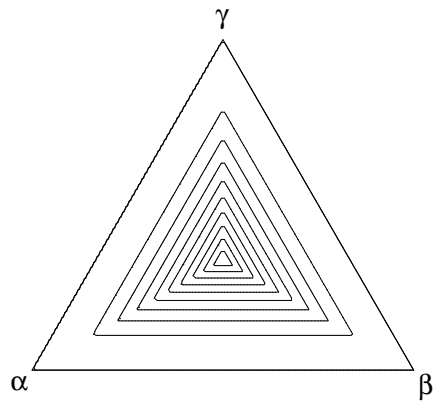

(a)

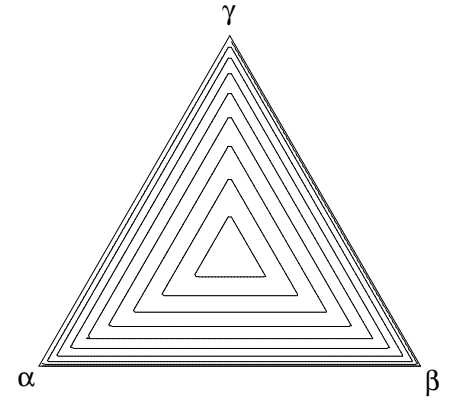

(b)

Figure 4. Representation in the ARR of a quality measure. Contour levels range form 0 to 1 with an increment of 0.1 (a) $\tilde{q}=q_{\alpha_{\min }}^{2}$, (b) $\tilde{q}=\sqrt{q_{\alpha_{\min }}}$.

It is important to note that, on one hand, every quality measure defines the shape of its
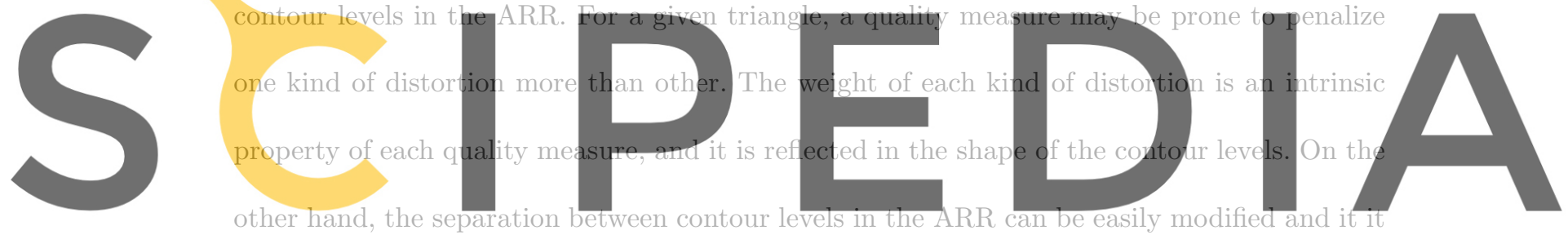

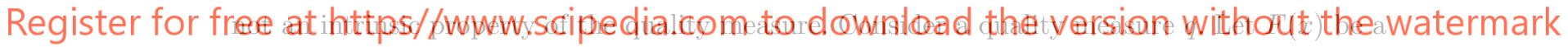

monotone function that maps $[0,1]$ into $[0,1]$. Then $\tilde{q}=F(q)$ is another quality measure whose contour levels have the same shape as those of $q$. However, the separation between contour levels is different.

For instance, compare Figure 3.a where $q_{\alpha_{\min }}$ is used with in figure 4.a and 4.b, where the quality measures are $\tilde{q}=q_{\alpha_{\min }}^{2}$ and $\tilde{q}=\sqrt{q_{\alpha_{\min }}}$ respectively. Notice that in figure 4.a the contour levels are concentrated near the center of the ARR and that in figure 4.b the contour levels are moved towards the boundary of the ARR. This may be of the major importance when a measure is used to optimize the quality of the mesh. 


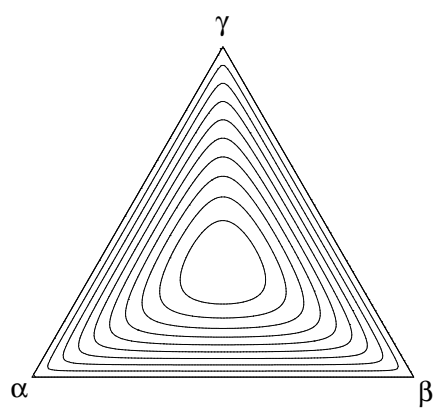

$q_{A L S}$

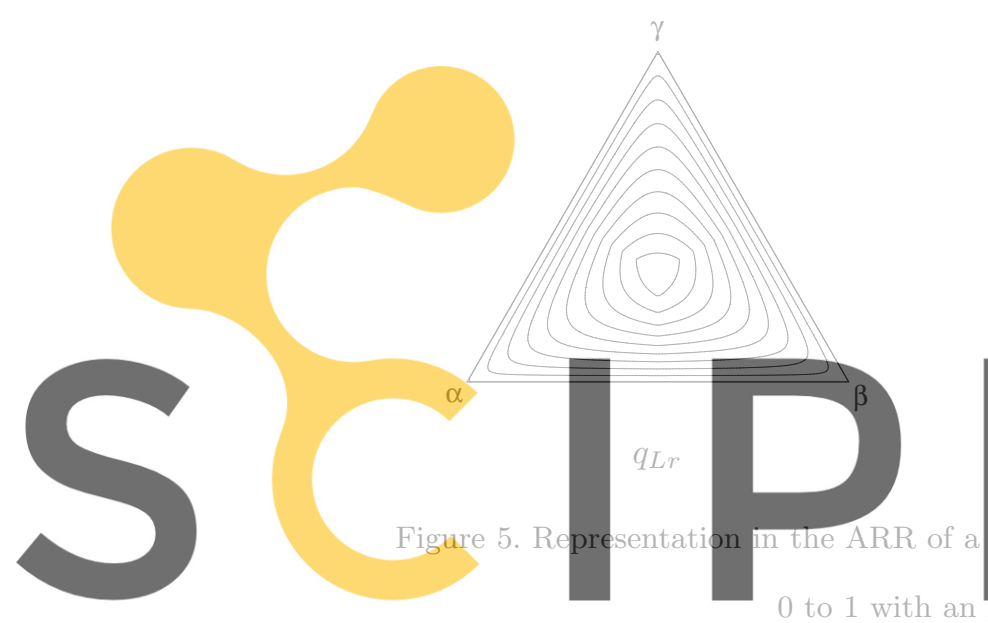

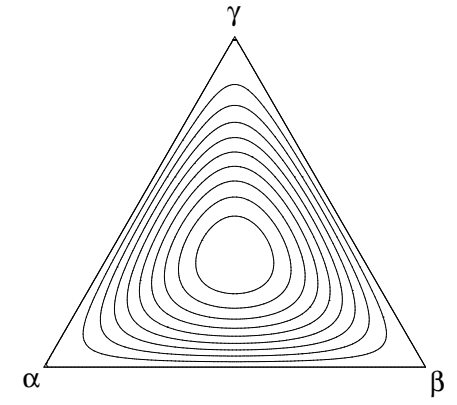

$q_{R r}$

\section{Register for free at https//www.scipedia.com to download the version without the watermark}

Figure 5 shows the contour levels of the measures $q_{A L S}, q_{R r}, q_{L r}$, and $q_{L h}$ in the ARR.

On one hand, the contour levels corresponding to $q_{A L S}$, and $q_{R r}$ have a similar shape. Both quality measures are almost constant near the center of the ARR (equilateral triangles), and the gradient of the contour levels increase significantly near its boundary (degenerate triangles). This behavior is more pronounced in the case of the $q_{A L S}$ measure. On the other hand, $q_{L r}$ and $q_{L h}$ have similar contour levels with a shape similar to an inverted triangle near the center of the ARR. Both measures present an almost linear variation of the contour level separation. Note, that $q_{L r}$ and $q_{L h}$ show a discontinuity in the slope of the contour levels for 
isosceles triangles. This property also appear in other quality measures that involves minimum or maximum values. Finally, it is important to note that the contour levels corresponding to the $q_{R r}$ measure are more separated near the vertices of the ARR. This reflects that this measure, compared with the others, is less sensible for triangles with an inner angle close to $\pi$.

\section{REPRESENTATION OF A OUALITY MEASURE OF MESHES}

Given a triangular mesh, it can also be represented in the ARR. Each element (triangle) will

be represented as a point in the ARR. In order to generate a coherent representation, the inner

angles of each triangle are sorted in a decreasing order, for instance: $\alpha \geq \beta \geq \gamma$. Note that
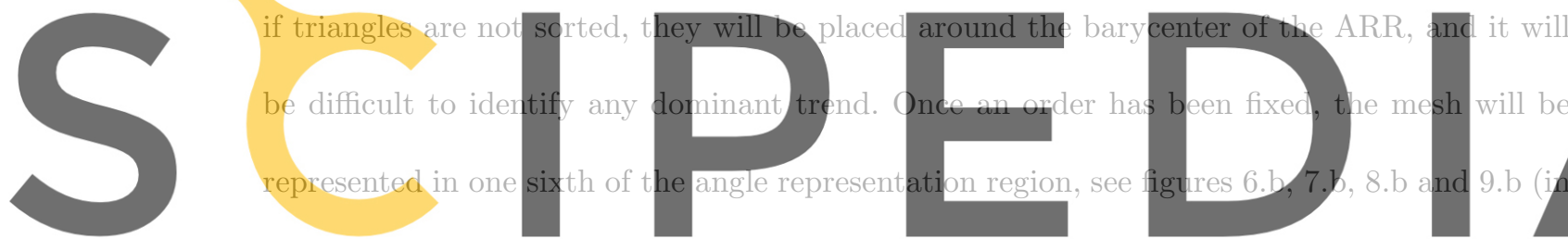

this figures point $\tilde{\alpha}_{r}$ is the intersection of the bisecting line $\overline{\alpha \alpha \prime}$ of figure $2 . \mathrm{b}$ and segment $\overline{\alpha_{r} \beta_{r}}$

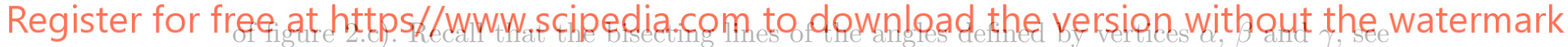

figure 2.b, split the ARR in six equivalent subregions.

The main objective here is to illustrate that the developed representation can be a useful and helpful tool if it is incorporated in a mesh generation software. To this end we will present four triangulations of a square domain obtained by different mesh generation algorithms. It is important to note that the developed representation clearly distinguishes them and contains, for instance, the information represented in histograms.

In order to gather all the information contained in the new representation of the mesh quality it is important to keep in mind the following six properties, which are well known by the finite 
element community comfortable with the area coordinates: 1 .- If the value of the biggest angle is $\pi$, then the triangle will be placed at vertex $\alpha$ (see figure 1.b). 2.- An equilateral triangle will be represented on vertex $O$ (see figure 2.a). 3.- The line $\overline{\tilde{\alpha}_{r} \beta_{r}}$ separates triangles with an obtuse inner angle and triangles with only acute inner angles (see figure 2.c). 4.- Isosceles triangles, $\alpha$ (the biggest angle) being the different angle, will be plotted on segment $\overline{\alpha O}$ (see figure 2.b). 5.- Isosceles triangles, $\gamma$ (the smallest angle) being the different angle, will be plotted on segment $\overline{\beta_{r} O}$ (see figure 2.b). 6.- Contour levels of any fair quality measure of triangles vary from the boundary of the $\operatorname{ARR}\left(q=0\right.$ on the segment $\left.\overline{\alpha \beta_{r}}\right)$, to vertex $O$ where they reach their maximum value $(q=1)$.

Figure 6.a shows the first triangulation of a square domain with high nodal density prescribed
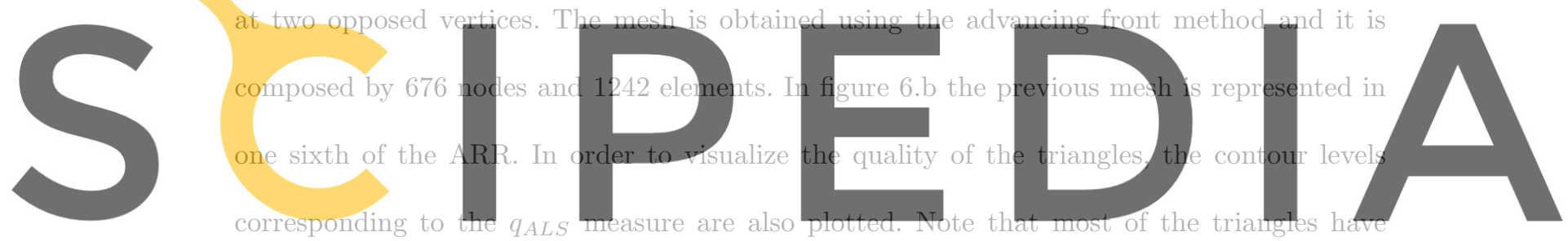

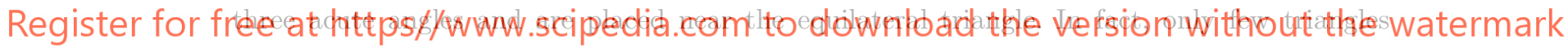

appears with the biggest angle greater than $\pi / 2$. Moreover, since most of them are plotted near the segment $\overline{\beta_{r} O}$, we can conclude that most of them are almost isosceles triangles being $\gamma$ (the smallest angle) the different angle. Therefore, all the elements of the mesh are well shaped triangles, $q_{A L S} \in(0.9,1.0)$. Moreover, there are no triangles with $q_{A L S} \leq 0.5$. It is important to note that this information is concentrated in only one figure and that it is in concordance with the histograms that shows the distribution of the quality of the triangles and the distribution of the biggest angle (figures 6.c and 6.d respectively).

Figure 7.a shows the triangulation of the same square domain when it is meshed using a non 


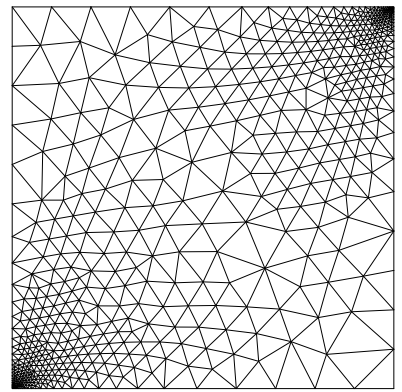

(a)

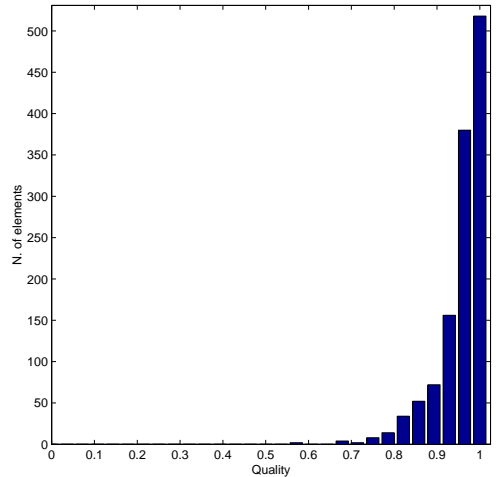

(c)

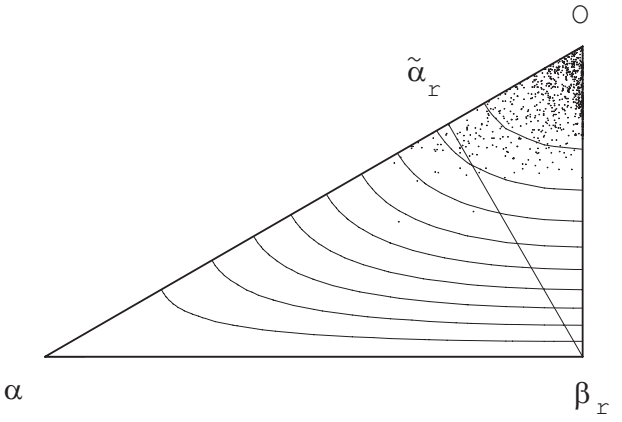

(b)

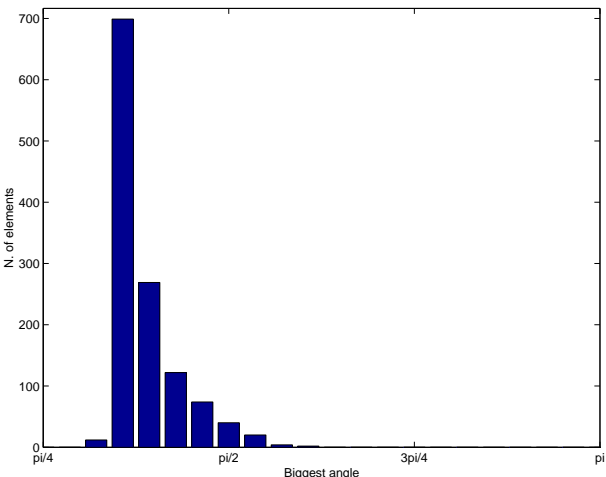

(d)

Figure 6. Representation of a meshes in the ARR: (a) triangular mesh, (b) contour levels of $q_{A L S}$, (c) distribution of the quality of the elements, (d) distribution of the biggest angle of the elements.

isotropic element size. The mesh is composed by 754 nodes and 1368 elements. Figure $7 . b$ plots the triangles on the ARR, also superposed are contour levels corresponding to $q_{A L S}$. Note that this representation points out that this mesh lacks of equilateral triangles. In fact, there are far less triangles with all three acute angles than in the previous case. A sparse distribution of the triangles in the $\mathrm{ARR}$ is obtained. It is important to note that $q_{A L S} \in(0.4,0.8)$. In particular, there is a triangle, which is almost isosceles, with an obtuse angle around $\alpha \approx 8 \pi / 9$ and $q_{A L S} \approx 0.05$. As in the previous example, these characteristics of the generated mesh are 


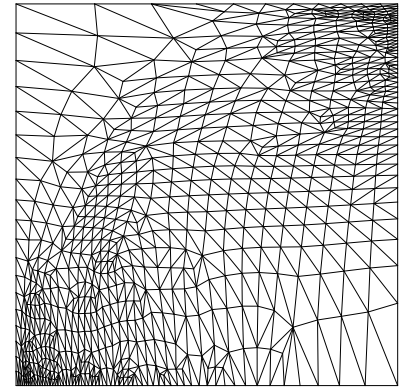

(a)

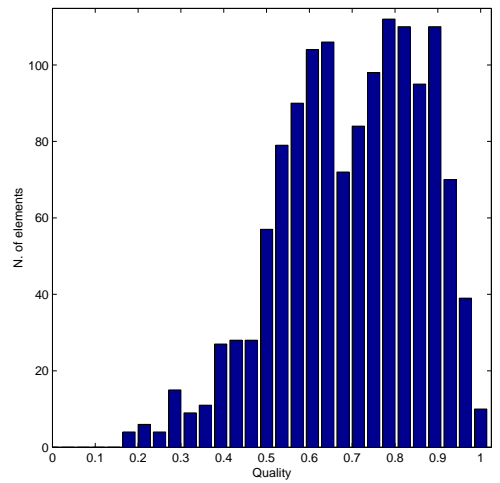

(c)

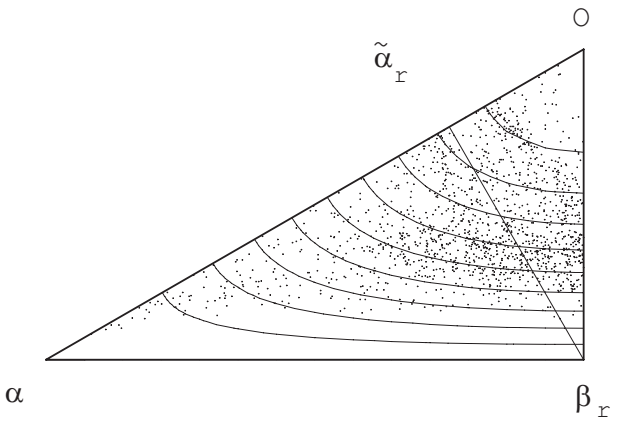

(b)

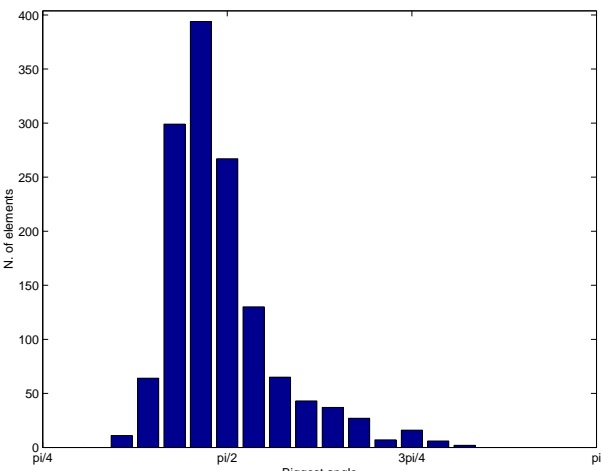

(d)

Figure 7. Representation of a meshes in the ARR: (a) triangular mesh, (b) contour levels of $q_{A L S}$, (c) distribution of the quality of the elements, (d) distribution of the biggest angle of the elements.

well represented in the ARR. The histograms of the distribution of the quality of the triangle and its biggest angle, figures 7.c and 7.d, corroborate this result.

Figure 8.a shows the third triangulation. The mesh is composed by 728 nodes and 1318 elements and it is obtained in two steps. First, quadrilaterals elements are generated [7]. Second, each quadrilateral is subdivided in two triangles joining the first and third nodes of the quadrilateral element. Figure 8.b shows the ARR representation and the contour levels of $q_{A L S}$. In this case most of the triangles are placed near the segment $\overline{\alpha O}$. Therefore, most of the 


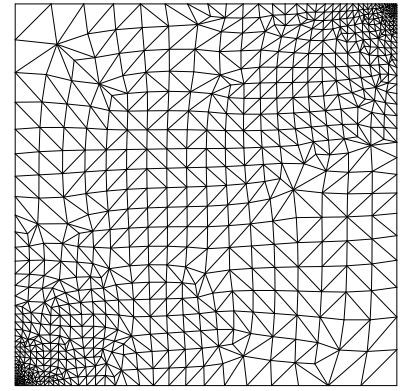

(a)

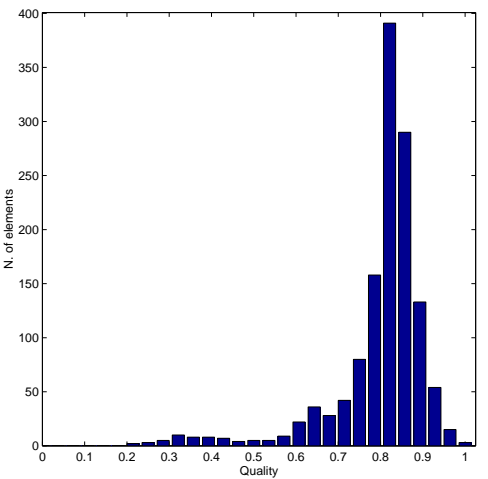

(c)

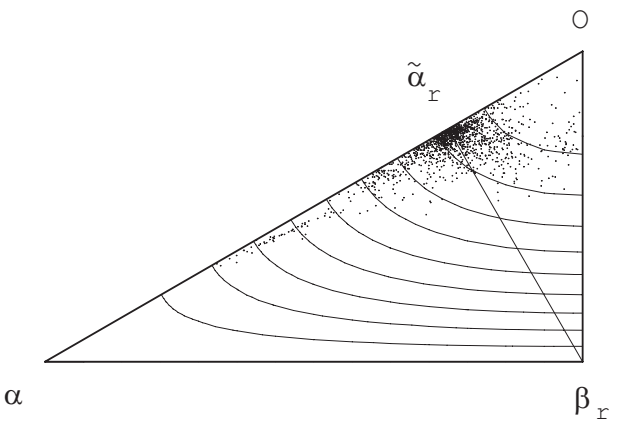

(b)

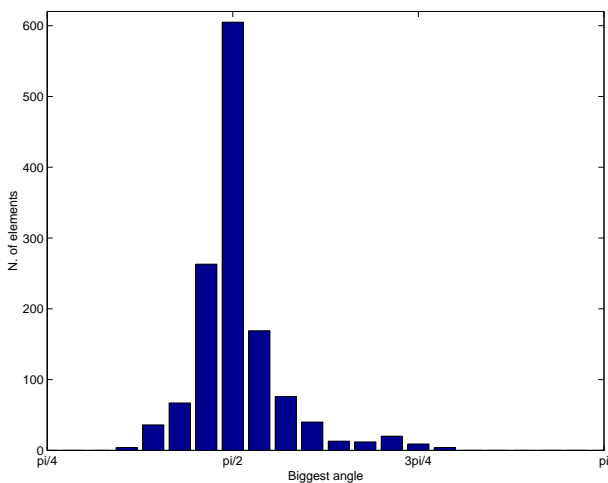

(d)

Figure 8. Representation of a meshes in the ARR: (a) triangular mesh, (b) contour levels of $q_{A L S}$, (c) distribution of the quality of the elements, (d) distribution of the biggest angle of the elements

triangles are isosceles with $\alpha$ being the different angle and largest angle. Moreover, the elements are concentrated in a cloud close to $\tilde{\alpha}_{r}$. Therefore, $\alpha$, the different angle, is approximately $\pi / 2$. Most of the triangles lie in the gap $q_{A L S} \in(0.8,0.9)$, and the lowest measure, $q_{A L S} \approx 0.2$, is associated to an almost isosceles triangle with $\alpha \approx 5 \pi / 6$. Again, this information is consistent with the histograms presented in figures 8.c and 8.d.

Finally, figure 9.a shows the fourth triangulation. This mesh is composed by the same 


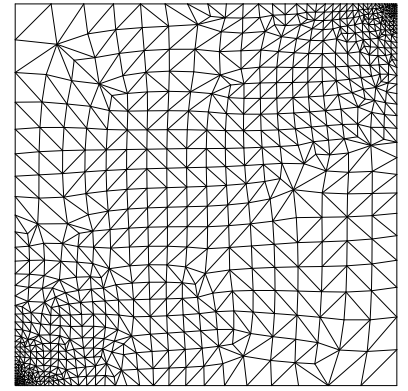

(a)

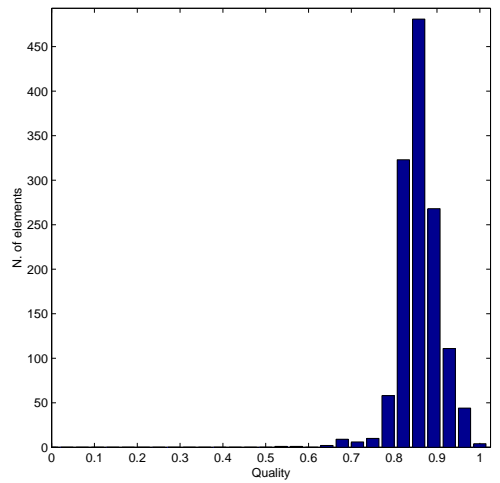

(c)

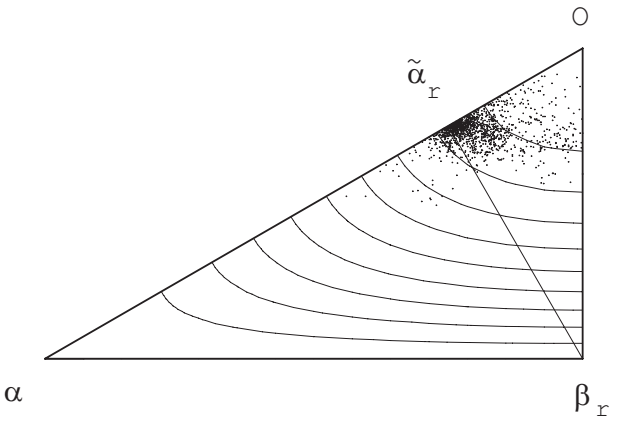

(b)

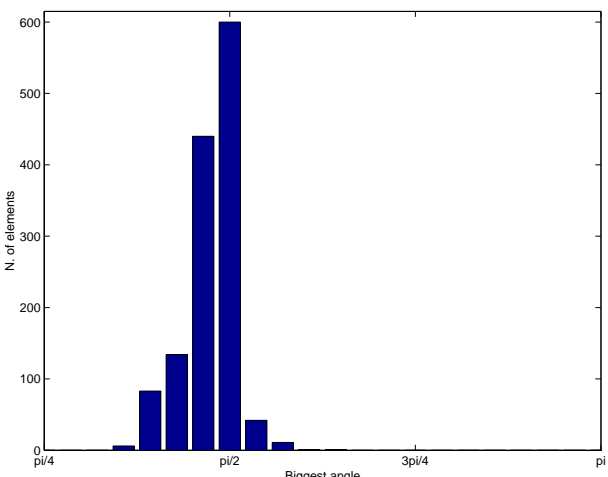

(d)

Figure 9. Representation of a meshes in the ARR: (a) triangular mesh, (b) contour levels of $q_{A L S}$, (c) distribution of the quality of the elements, (d) distribution of the biggest angle of the elements.

728 nodes of the previous example. Connectivity is defined using a standard Delaunay triangulation. Figure 9.b shows the ARR representation and $q_{A L S}$. The cloud of triangles is now more concentrated and closer to vertex $O$ because Delaunay favors equilateral triangles. Therefore, the quality of the triangles also improves. This behavior can be also observed in the histograms presented in figures 9.c and 9.d. 


\section{CONCLUSIONS}

This paper proposed a new procedure to represent the quality measure for triangles. It identifies each family of triangles by the area coordinates. They are represented in a bounded domain called the angle representation region. Basic regular triangles, such as equilateral and isosceles triangles, can be easily identified in the angle representation region. Moreover, since degenerate triangles lies on its boundary, the new representation clearly distinguish them. Contour levels corresponding to different quality measures can be plotted easily using this representation. This new procedure can also be used in order to graphically represent triangular meshes in the angle representation region. Insightful conclusions can be gained about contours depicting quality measures and about quality measures of specific triangles or meshes, in particular for the finite element community familiar with area coordinates.

\section{REFERENCES}

1. George PL, Borouchaki H. Delaunay Triangulation and meshing. Application to finite elements. Hermes, Paris, 1998.

2. Thompson JF, editor. Handbook of Grid Generation. CRC Pres, 1999

3. Baker TJ. Element quality in tetrahedral meshes. In Proceedings of the 7th International Conference on Finite Element Methods in FLow Problems, 1989; Huntsville, USA.

4. Field DA. Qualitative measures for initial meshes. International Journal for Numerical Methods in Engineering 2000; 47 887-906.

5. Parthasarathy VN, Graichen CM, Hathaway AF. A comparision of tetrahedron quality measures. Finite Elements in Analysis an Design 1993; 15, 255-261.

6. Zienkiewicz OC, Taylor RL. The finite Element Method. Volume 1: The Basis Butterworth-Heinemann, 2000.

7. Sarrate J, Huerta A. Efficient unstructured quadrilateral mesh generation. International Journal for 
Numerical Methods in Engineering 2000; 49, 1327-1350. 